\title{
Linear Ridge Arrays Induce a Self-Cleaning Functionality and Improved Electrochemical Performance during the Oxygen Evolution Reaction
}

\author{
Taylor, K. Audrey; ${ }^{1}$ Muo, Tiffany; ${ }^{1}$ Sonea, Ana; ${ }^{1}$ Yee, B. Brenden; ${ }^{1}$ Chen, Jiayue; ${ }^{1}$ and Gates, D. \\ Byron $^{1 *}$ \\ ${ }^{1}$ Affiliations: Simon Fraser University, Department of Chemistry, \\ 8888 University Drive, Burnaby, BC V5A 1S6 \\ bgates@sfu.ca.
}

\section{Extended Abstract}

Electrocatalytic water splitting on an industrial-scale for chemical energy storage through hydrogen gas production will require further improvements to the other half-reaction, the oxygen evolution reaction (OER). This half-reaction has a high kinetic barrier due to superoxide bond formation, and four proton-coupled electron transfer steps. Considerable attention has focused on improving the reaction kinetics of the OER by tuning the electronic structure of electrocatalysts. Gas management is, however, still a problematic factor for industrial-scale water splitting and especially for highly active electrocatalysts. Accumulation of bubbles on electrode surfaces can result in blocked active sites and increased resistances. Recent literature has pointed to the importance of the architecture of the electrode surfaces for their influence on effectively releasing bubbles without the need for additional energy input (e.g., high shear flows or higher overpotentials). Electrodes with nanoscale textures have demonstrated superaerophobic qualities with a high degree of wetting during electrocatalytic gas evolving reactions. Higher reactions rates can also be achieved using microscale geometries that can assist in the removal of gas from surfaces. These lessons can be applied to a variety of gas evolving reactions, but are of particular interest here for the OER.

Herein, we prepared regular arrays of linear ridges with well-defined dimensions and microscale separations between these features. The influence of feature spacing was systematically studied for a series of electrodes and correlated to the performance of the OER. The mass transfer processes associated with the gas evolution were investigated using a high-speed camera. Characterization of the adhered bubbles enabled the use of empirical methods to determine the volumetric flux of product gas, and the bubble residence times. The linear ridges promoted a self-cleaning effect as one bubble would induce neighbouring bubbles to simultaneously release from the electrode surfaces. The ridges provided preferential bubble growth sites and expedited a synchronous detachment of bubbles with similar diameters. Linear ridges with a separation distance of $200 \mu \mathrm{m}$ achieved nearly a two-fold increase in current density relative to the planar electrode at high operational potentials. Comparison of this series of electrodes with their tuned spacing of the microscale, linear features provided a systematic correlation between feature separation and their gas evolution efficiency. 